\title{
Autopercepção da saúde bucal de ídosos
}

\author{
- Simone Kreve Faculdade de Odontologia de Ribeirão Preto, Universidade de São Paulo (USP), São Paulo, SP, Brasil \\ - Gabriela Caroline D’Avila Universidade do Oeste do Paraná (Unioeste), Cascavel, PR, Brasil • Letícia Oliveira Santos \\ Universidade Paranaense (Unipar), Cascavel, PR, Brasil • Andréa Cândido dos Reis Faculdade de Odontologia de \\ Ribeirão Preto, Universidade de São Paulo (USP), São Paulo, SP, Brasil
}

RESUMO | Objetivo: Avaliou-se a autopercepção das condições de saúde bucal de um grupo de idosos por meio da aplicação do índice General Oral Health Assessment Index (Gohai). Métodos: Participaram do estudo 136 idosos, frequentadores do Centro de Revitalização da Terceira Idade (Certi), onde aplicou-se um questionário com questões do índice Gohai sobre a autopercepção das condições bucais. Realizou-se análise descritiva do resultado de cada uma das perguntas. Resultados: Dos idosos entrevistados, 78,7\% responderam sempre sentir dor ou desconforto ao deglutir; 55,1\% relataram desconforto em comer algum alimento, e 66,9\% relataram insatisfação com a aparência da sua boca. A média global do índice Gohai quando aplicada à escala de interpretação foi de 28,88, classificada com uma percepção de saúde bucal "ruim" sobre a qualidade de vida. Conclusão: Os idosos avaliaram negativamente suas condições bucais pelo desconforto em comer, dificuldade/dor para deglutir e insatisfação com sua condição bucal.

ABSTRACT | Self-perception of oral health in older adults - Aim: The oral health self-perception conditions of older adults was analyzed by applying General Oral Health Assessment Index (Gohai). Methods: A total of 136 participants over 60 years members of the Center for Revitalization of Older Adults (CERTI) were assessed by the Gohai questionnaire on oral self-perception conditions was applied. Descriptive analysis was performed on the results. Results: Of all participants, $78.7 \%$ mentioned always feeling pain or discomfort while swallowing; $55.1 \%$ reported discomfort while eating; and $66.9 \%$ reported dissatisfaction with the appearance of their mouth. The overall average of the Gohai Index when applied to the interpretation scale for this index was 28.88, classified as a "bad" oral health condition. Conclusion: The participants negatively evaluated their oral condition due to discomfort in eating, difficulty/pain to swallow and dissatisfaction with their oral condition.

\section{AUTOR CORRESPONDENTE}

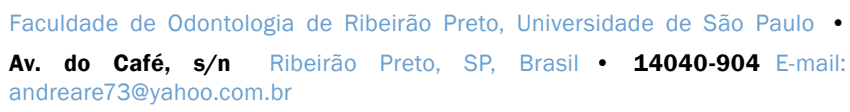
Faculdade de Odontologia de Ribeirão Preto, Universidade de São Paulo • Av. do Café, s/n Ribeirão Preto, SP, Brasil • 14040-904 E-mail: andreare73@yahoo.com.br

Cândido dos Reis Departamento de Materials Dentarios e Prótese,

- Recebido 07 ago. 2019 • Aceito 07 out. 2019

- DOI: http://dx.doi.org/10.11606/issn.2357-8041.clrd.2020.160816 


\section{INTRODUÇÃO}

O interesse no envelhecimento saudável é crescente $^{1,2}$ e, a partir do momento que o idoso se torna incapaz de participar plenamente das atividades desejadas, a qualidade de vida é afetada. ${ }^{3,4}$

Alterações nas condições sistêmicas, bem como seus tratamentos, tornam-se mais prevalentes com o envelhecimento, ${ }^{1,5}$ e influenciam nas expectativas e no modo de conduzir a vida. ${ }^{3}$ A satisfação pessoal está relacionada a fatores sociodemográficos, estilo de vida e saúde, ${ }^{6,7}$ e incluem a prática de atividades educativas, utilização da criatividade, contato com crianças e atividades sociais. $^{8}$

Com o avanço da idade surgem também os efeitos negativos sobre a saúde geral e a integridade dos tecidos orais, como dentes, mucosas ou músculos.9-11 Doenças como a diabetes mellitus e a ingestão de um grande número de medicamentos podem reduzir o fluxo salivar, ${ }^{10} \mathrm{e}$ este fator pode acentuar a presença de cárie dentária e doença periodontal. ${ }^{12} \mathrm{~A}$ deterioração da saúde bucal também é um agravante para doenças cardiovasculares e respiratórias infecciosas, endocardite, infecções articulares e, dentre outras, abscessos cerebrais. ${ }^{1,11,13}$

A perda dentária é responsável por modificar a fisiologia normal, promove mudanças na aparência; redução da altura facial; protrusão do lábio inferior e do queixo; deficiências funcionais e sensoriais da mucosa oral e da musculatura; afeta a fala; a função mastigatória,5,9,10,14 causando dor e desconforto; ${ }^{15}$ abaixa a autoestima; sendo esses fatores contribuintes para uma autopercepção negativa da sua saúde oral. ${ }^{9,11}$ A perda de dentes não é uma consequência normal do envelhecimento, mas sim uma culminação de eventos ao longo da vida, ${ }^{16}$ e isso limita a participação em atividades sociais, reduz a autoconfiança, causa um envelhecimento prematuro, altera a autoimagem, e reduz a socialização. ${ }^{11,15}$ Além disso, a ausência dentaria influencia na nutrição. Cabe lembrar que as características físicas, fisiológicas, psicossociais e emocionais dos idosos os identificam como um grupo populacional de alto risco para má nutrição. ${ }^{9,12}$ Uma dieta pouco saudável, ${ }^{11}$ frequentemente monótona, com baixo teor de energia e deficiências de nutrientes, retinol, hidratos de carbono e ácido fólico ${ }^{17}$ levam a deficiências funcionais, declínio da força muscular e fragilidade. ${ }^{5,18}$

A autopercepção de saúde é considerada um bom indicador do estado de saúde, pois incorpora componentes físicos, cognitivos e emocionais..,19 Atualmente, os conceitos de saúde bucal são voltados a uma perspectiva psicossocial, a medidas qualitativas e à incorporação do ponto de vista do paciente, bem como o efeito das doenças bucais no seu bem-estar funcional e psicológico. ${ }^{2}$

Pela importância desses fatores e sua influência na qualidade de vida e longevidade da população idosa, selecionamos o questionário Geriatric Oral Health Assesment Index (Gohai) para analisar a situação atual da população idosa, que no estudo foi representada por 136 idosos frequentadores do Centro de Revitalização da Terceira Idade (Certi). O objetivo foi analisar a autopercepção de saúde bucal verificada a partir da aplicação do questionário.

\section{MATERIAIS E MÉTODOS}

Este estudo descritivo transversal foi realizado com 136 indivíduos idosos que participam das atividades diárias do Certi na cidade de Toledo/PR.

Os critérios de inclusão foram: idosos de ambos os sexos com idade maior ou igual a 60 anos, funcionalmente independentes, que aceitassem participar voluntariamente do estudo.

Como critérios de exclusão: idosos com doença mental que incapacitasse a resposta ao questionário, recusa em assinar o termo de consentimento livre e esclarecido, e analfabetos.

Os dados foram coletados por meio do questionário Gohai modificado ${ }^{20}$ que é composto por doze perguntas 
objetivas em que o idoso tem como opções as respostas "sempre", "às vezes" e "nunca”. As doze situações correspondem a três funções elementares: física (alimentação, fala, deglutição), psicológica e presença de dor ou desconforto.

O nível de mensuração desses itens é categórico, de forma que às respostas "sempre", "às vezes" e "nunca" foram atribuídos pesos 1, 2 e 3, respectivamente. As perguntas de números são computadas inversamente às demais. Para obtenção do índice global realiza-se a soma simples dos escores, numa escala de doze a 36 , sendo que a autopercepção pode ser classificada em "ótima" (34 a 36 pontos), "regular" (31 até 33 pontos) e "ruim" (menos 30 pontos).
Os dados foram analisados segundo a estatística descritiva.

Este estudo foi iniciado após aprovação pelo Comitê de Ética em Pesquisa em Seres Humanos (CAAE 54581316.1.oooo.0109) e após obtenção do Termo de Consentimento Livre e Esclarecido assinado pelos voluntários convidados.

\section{RESULTADOS}

A média global do índice Gohai foi de 28,88 $(\mathrm{DP}=2,81)$. Essa pontuação, quando aplicada à escala de interpretação para o índice, representa uma classificação da percepção de saúde bucal considerada "ruim" sobre a qualidade de vida dos idosos (Tabela 1).

Tabela 1 | Distribuição de frequências e medidas de tendência central do índice Gohai

\begin{tabular}{|c|c|c|c|c|}
\hline \multicolumn{5}{|c|}{ Descritivos } \\
\hline & & & Estatística & Erro \\
\hline \multirow[t]{12}{*}{ Índice Gohai } & \multicolumn{2}{|l|}{ Média } & 28,88 & 0,240 \\
\hline & \multirow{2}{*}{$\begin{array}{l}\text { 95\% intervalo de } \\
\text { confiança para média }\end{array}$} & Limite inferior & 28,40 & \\
\hline & & Limite superior & 29,35 & \\
\hline & \multicolumn{2}{|l|}{$5 \%$ da média aparada } & 29,02 & \\
\hline & \multicolumn{2}{|l|}{ Mediana } & 29,00 & \\
\hline & \multicolumn{2}{|l|}{ Variância } & 7,829 & \\
\hline & \multicolumn{2}{|l|}{ Erro desvio } & 2,798 & \\
\hline & \multicolumn{2}{|l|}{ Mínimo } & 20 & \\
\hline & \multicolumn{2}{|l|}{ Máximo } & 34 & \\
\hline & \multicolumn{2}{|l|}{ Amplitude interquartil } & 4 & \\
\hline & \multicolumn{2}{|l|}{ Assimetria } & $-0,737$ & 0,208 \\
\hline & \multicolumn{2}{|l|}{ Curtose } & 0,094 & 0,413 \\
\hline
\end{tabular}

As questões que compõem o índice Gohai, bem como as respostas, estão descritas na sequência.

1. "Nos últimos meses você limitou o tipo ou a quantidade de alimentos que come devido a problemas com seus dentes ou próteses?”. Responderam "sempre" 7,4\% $(\mathrm{N}=10)$; "às vezes" $25,7 \%(\mathrm{~N}=35)$; e "nunca" 66,9\% ( $\mathrm{N}=91)$.

2. "Teve problema em mastigar alimentos como carne sólida ou maçã?" Responderam "sempre"
$15,4 \%(\mathrm{~N}=21)$; “às vezes" 20,6\% ( $=28)$; e "nunca" $64 \%(\mathrm{~N}=87)$.

3. "Foi capaz de engolir confortavelmente?". Responderam "sempre" $2,9 \%(\mathrm{~N}=4)$; "às vezes" $18,4 \%(\mathrm{~N}=25)$; e "nunca" $78,7 \%(\mathrm{~N}=107)$.

4. "Mudou o jeito de falar por causa dos problemas da sua boca?" Responderam "sempre" 13,23\% ( $\mathrm{N}=18)$; "às vezes" 21,32\% ( $=29)$; e "nunca" $65,44 \%(\mathrm{~N}=89)$. 
5. "Foi capaz de comer qualquer coisa sem sentir desconforto?". Responderam "sempre" 22,1\% $(\mathrm{N}=30)$; "às vezes" 22,8\% ( $=31)$; e "nunca" $55,1 \%(\mathrm{~N}=75)$.

6. "Deixou de se encontrar com outras pessoas por causa de sua boca?". Responderam "sempre" $3,7 \%(\mathrm{~N}=5)$; "às vezes" $8,1 \%(\mathrm{~N}=11)$; e "nunca" $88,2 \%(\mathrm{~N}=120)$.

7. "Sentiu-se feliz ou satisfeito com a aparência de sua boca?". Responderam "sempre" 16,9\% ( $\mathrm{N}=23)$; "às vezes" $16,2 \%(\mathrm{~N}=22)$; e "nunca" $66,9 \%(\mathrm{~N}=91)$.

8. "Teve que tomar remédio para passar a dor ou desconforto de sua boca?". Responderam "sempre" 7,4\% ( $=10)$; "às vezes" $27,9 \%(\mathrm{~N}=38)$; e "nunca" 64,7\% ( $=88)$.

9. "Preocupou-se ou teve cuidados com seus dentes, gengivas ou próteses?". Responderam "sempre" $5,1 \%(\mathrm{~N}=7)$; "às vezes" $22,1 \%(\mathrm{~N}=30)$; e "nunca" 72,8\% ( $\mathrm{N}=99)$.

10."Chegou a sentir nervoso por causa de problemas na sua boca"? Responderam "sempre" $5,1 \%(\mathrm{~N}=7)$; "às vezes" 25,7\% ( $=35)$; e "nunca" $69,1 \%(\mathrm{~N}=94)$.

11."Evitou comer junto com outras pessoas por causa dos problemas com sua boca?”. Responderam "sempre" 1,5\% ( $=2)$; "às vezes" 6,6\% $(\mathrm{N}=9)$; e "nunca" $91,9 \%(\mathrm{~N}=125)$.

12."Teve sensibilidade nos dentes ou gengivas ao contato com calor, frio ou doces?”. Responderam "sempre" 24,3\% ( $\mathrm{N}=33)$; "às vezes" $31,6 \%(\mathrm{~N}=43)$; e "nunca" 44,1\% ( $=60)$.

\section{DISCUSSÃO}

O Gohai simplificado utilizado neste artigo foi desenvolvido por Atchison e Dolan ${ }^{21}$ para pesquisas com idosos norte-americanos, e foi traduzido e validado para ser aplicado à população idosa brasileira ${ }^{20,21}$, tanto em estudos epidemiológicos quanto na prática clínica diária,4,22,23 tendo como forma de mensuração a autopercepção. ${ }^{2,5,24} \mathrm{O}$ índice é composto por doze perguntas fechadas e sua formulação é baseada em três aspectos, físico (alimentação, fala, deglutição), psicológico (preocupação ou cuidado com a própria saúde bucal, insatisfação com a aparência, autoconsciência relativa à saúde bucal, e o fato de evitar contatos sociais devido a problemas odontológicos) e presença de dor ou desconforto (uso de medicamentos para aliviar estas sensações, desde que provenientes da boca).,19

Este estudo analisou cada uma das respostas obtidas a partir da entrevista de 136 idosos.

A primeira pergunta questionou sobre limitar a quantidade, ou ainda mudar o tipo de alimento por causa dos dentes, e 66,9\% dos entrevistados responderam que não sentem essa necessidade, $25,7 \%$ responderam que às vezes necessitam alterar/limitar o tipo de alimento. De acordo com outro estudo, esses foram os problemas bucais mais relatados. ${ }^{2}$ Nem sempre as escolhas alimentares estão relacionadas às preferências, ${ }^{25}$ uma vez que a necessidade de um maior número de golpes mastigatórios torna a moagem dos alimentos menos eficiente ${ }^{26}$ e o resultado pode ser a alteração da dieta de uma contendo fibras e proteínas para uma contendo mais carboidratos, que requer menos esforços mastigatórios. ${ }^{10}$

De acordo com a pergunta sobre problemas em mastigar, 64\% responderam não ter problemas. A efetividade na mastigação depende da dentição, da articulação temporomandibular e dos músculos orofaciais, e geralmente é reduzida em associação ao envelhecimento, como resultado da redução da massa muscular. ${ }^{9,10,27}$ Foi relatado que as dificuldades de mastigação alimentar têm um impacto maior na qualidade de vida do indivíduo do que em seu estado nutricional. ${ }^{28}$

A manutenção dos dentes e suporte oclusais possibilitam uma dieta diversificada, ${ }^{29}$ pois declínios no contato oclusal posterior, força oclusal diminuída, e taxa de fluxo salivar foram associados à redução 
do desempenho mastigatório em idosos, ${ }^{26}$ além da perda funcional que pode levar a alterações ósseas e musculares severas, interferindo em condições sistêmicas como disfunção temporomandibular e distúrbios do sono. ${ }^{15,30}$

A pergunta 3 questiona sobre dificuldade na deglutição, e 78,7\% dos entrevistados descreveu ter dificuldade. Esse item foi desenvolvido para medir os problemas que as pessoas com xerostomia podem encontrar, ${ }^{10,12,23}$ uma vez que a falta de lubrificação dificulta a deglutição. ${ }^{9}$ Tal desconforto também pode ser resultado de perdas dentárias, por redução na dimensão vertical e no espaço da cavidade bucal que interfere no movimento da língua. ${ }^{31}$ Já a xerostomia pode surgir pelo uso de drogas xerogênicas, fatores psicológicos, doenças autoimunes, diabetes ou radioterapia..$^{10,23}$

Sobre mudar o "jeito de falar por causa de problemas na boca”, 65,44\% dos entrevistados responderam não sofrer desse tipo de problema. Já outros autores observaram que a maior parte do desconforto na fala foi relatado por usuários de prótese, principalmente os usuários de prótese total bimaxilar. ${ }^{32} \mathrm{O}$ envelhecimento por si só é um fator importante no desenvolvimento de distúrbios de voz ${ }^{33}$ pois a cartilagem e os músculos envolvidos na fonação também sofrem alterações estruturais, na intensidade e na qualidade vocal, ${ }^{34} \mathrm{e}$ esses são fatores que afetam negativamente a qualidade de vida. ${ }^{33}$

A pergunta 5 questiona sobre sentir desconforto em comer algum alimento, 55,1\% dos idosos responderam que "sempre" e 22,8\% responderam que "às vezes" sentem algum desconforto. O envelhecimento causa prejuízo no apetite, pois reduz o paladar e o olfato, o que torna o alimento menos atraente. ${ }^{35}$ Essas mudanças alteram os hábitos alimentares e reduzem a disponibilidade e a absorção de nutrientes, o que pode levar a deficiências nutricionais e vários problemas de saúde, incluindo debilidade no sistema imunológico, aumento no risco de infecções, dificuldade na cicatrização de feridas e fraqueza muscular. ${ }^{35}$ De acordo com Silva, Demarco e Feldens,${ }^{36}$ a dificuldade em comer foi um dos itens mais reportados, e além de uma dieta pobre em nutrientes, o desconforto pode estar relacionado ao uso de próteses. ${ }^{9}$ Entretanto, Amagai et al. ${ }^{37}$ relataram que a reabilitação oral de idosos com próteses totais, associada a aconselhamento dietético aumentou a ingestão de alimentos saudáveis e melhorou a qualidade de vida.

Quando questionados sobre socialização, 88,2\% responderam não deixar de encontrar outras pessoas por causa de sua boca. Idosos entrevistados por outro estudo ${ }^{38}$ relataram a importância da saúde bucal para o bem-estar, que incluiu manter contatos sociais, sorrir, ter hálito fresco e a necessidade de sentir-se socialmente seguro tanto na comunicação quanto na alimentação. Já a incapacidade funcional e social, a dor, e o desconforto psicológico foram situações mais frequentemente relatadas por pacientes portadores de prótese total bimaxilar. ${ }^{32}$

A pergunta 7 questiona sobre a satisfação com a aparência da boca, e nesse quesito $66,9 \%$ responderam não estar satisfeitos. De acordo com Osman, Khalifa e Alhajj, ${ }^{2}$ o quesito preocupação com a aparência foi frequentemente relatado entre a população idosa. A estética facial e a aparência física têm grande potencial em afetar a vida social. ${ }^{39}$ Existe uma série de mudanças que ocorrem na dentição que são consideradas parte do envelhecimento normal, como desgaste, lascamento e aparecimento de linhas de fratura no esmalte, exposição de dentina, deposição de dentina secundária, afinamento do esmalte e, dentre outras, acúmulo de manchas na superfície. ${ }^{10} \mathrm{~A}$ autopercepção da aparência facial está frequentemente relacionada à preocupação com opiniões de outras pessoas, e nesse contexto os lábios e dentes são duas características que definem a aparência geral do rosto. ${ }^{39}$

A respeito da pergunta que envolve uso de medicação para desconforto relacionado à boca, 64,7\% responderam nunca fazer uso de medicação, 
corroborando resultados de outros autores. ${ }^{2}$ Idosos tendem a referir problemas de saúde quando experimentam condições dolorosas ou limitantes, e nesse sentido é comum uma autopercepção negativa da sua saúde bucal. ${ }^{40}$ Os problemas mais descritos são: xerostomia, ardência bucal, halitose, alterações de tecidos moles, saburra lingual, desordens temporomandibulares, e retração gengival.2,10,23

Quando questionados sobre sentir preocupação com algum problema da boca, $72,8 \%$ responderam que não; e sobre sentir nervosismo por problemas da boca, 69,1\% também responderam que não. O contrário foi relatado por Echeverria et al., ${ }^{41}$ no qual o desconforto psicológico ("preocupado por causa de seus dentes, boca ou dentaduras") foi a dimensão com o maior impacto na qualidade de vida relacionada à saúde bucal nas avaliações inicial e de acompanhamento.

A pergunta 11 questiona sobre evitar se alimentar junto de outras pessoas e 91,9\% responderam que não se sentem constrangidos. Considerando esse aspecto, idosos portadores de próteses podem ter maior dificuldade de adaptação cognitiva e neuromuscular, ${ }^{9}$ dificultando a adaptação. Sentir vergonha pode ser um componente importante para o desenvolvimento e a manutenção de transtornos alimentares, ${ }^{42}$ visto que sintomas orais estão relacionados com desnutrição, números mais elevados de comorbidades, dependência física e sintomas gastrintestinais, além de comerem menos e sozinhos. ${ }^{43} \mathrm{~A}$ disfunção oral também pode ser resultado dos muitos eventos negativos que acompanham o envelhecimento, como doença ou lesão física, morte do cônjuge, realocação, e o estresse psicológico. ${ }^{44}$

Quando os idosos foram questionados sobre a sensibilidade de seus dentes ou gengivas no contato com calor, frio ou doces, a maioria $(44,1 \%)$ respondeu que não sente esse tipo de alteração, e 31,6\%, responderam que às vezes sentem seus dentes ou gengivas sensíveis. A destruição periodontal por si só não está especificamente associada ao envelhecimento, entretanto uma perda de inserção periodontal é normal, ${ }^{10}$ expondo a raiz dentária e causando certa sensibilidade. A periodontite pode afetar a qualidade de vida por ser uma condição inflamatória que afeta os tecidos de sustentação dos dentes. ${ }^{45}$ Os fatores de risco são os mesmos em adultos jovens e idosos, embora possam ser mais proeminentes em indivíduos mais velhos, ${ }^{13}$ que podem ser menos capazes de remover depósitos de biofilme como resultado de destreza reduzida, e diminuição da acuidade visual. ${ }^{10}$ Portanto a doença periodontal descontrolada ao longo da vida tem um impacto maior em pacientes idosos comparado a pacientes jovens, ${ }^{13}$ além de estar relacionada à etiologia de várias doenças sistêmicas relevantes. ${ }^{13}$ As bactérias orais podem causar infecção de órgãos distantes (por exemplo, endocardite, abscesso pulmonar e pneumonia). ${ }^{13} \mathrm{~A}$ fim de prevenir condições médicas, é importante que os cuidados periodontais sejam eficazes, sobretudo em idosos frágeis, hospitalizados, sob cuidados de longa duração, pacientes em unidades de diálise e aqueles com demência. ${ }^{13}$

No grupo estudado a autopercepção de saúde bucal foi "ruim" a partir do índice Gohai médio encontrado $(28,88 \pm 2,79)$. Este valor esteve fortemente associado às perguntas que envolveram satisfação com sua condição bucal, dificuldade de engolir e desconforto em comer alguns alimentos. Em relação à dificuldade de engolir, ficam evidentes os transtornos causados pela xerostomia. A redução do paladar e do olfato em virtude do envelhecimento, as dificuldades com ausências dentárias e uso de próteses, redução na força muscular, na inserção periodontal, entre outros, são fatores que associados prejudicam uma dieta variada e suficiente ingestão de nutrientes, e consequentemente esses fatores somados são responsáveis por gerar uma insatisfação com sua condição bucal. Esse levantamento nos faz vislumbrar a necessidade de maiores investimentos em políticas públicas voltadas para conscientização do autocuidado, englobando aspectos emocionais, 
culturais, e físicos visando reduzir as doenças associadas ao envelhecimento, bem como o alto custo que os procedimentos de saúde acarretam, o que contribuirá significativamente para a melhora da qualidade de vida dessa população.

\section{CONCLUSÃO}

Os achados mostram uma autopercepção em saúde oral considerada "ruim" de acordo com o índice Gohai. Os principais fatores que levaram a esse resultado foram o desconforto em comer algum alimento, dificuldade de deglutição e insatisfação com sua condição bucal, mostrando uma interrelação entre as três funções elementares: física, psicológica e presença de dor ou desconforto na qualidade de vida dos idosos.

\section{REFERÊNCIAS}

1. Ghezzi EM, Ship JA. Systemic diseases and their treatments in the elderly: impact on oral health. J Public Health Dent. 2000;60(4):289-96. doi: https://doi.org/10.1111/ j.1752-7325.200o.tbo3337.x

2. Osman SM, Khalifa N, Alhajj MN. Validation and comparison of the Arabic versions of GOHAI and OHIP-14 in patients with and without denture experience. BMC Oral Health. 2018;18(1):157. doi: https://doi.org/10.1186/s12903-018-0620-5

3. Pistorius J, Horn JG, Pistorius A, Kraft J. Oral Health-Related Quality of Life in Patients with Removable Dentures. Schweiz Monatsschr Zahnmed. 2013;123:964-71.

4. Piuvezam G, Lima KC. Self-perceived oral health status in institutionalized elderly in Brazil. Arch Gerontol Geriatr. 2011;55(1):5-11. doi: https://doi.org/10.1016/j.archger.2011.04.017

5. Shwe PS, Ward SA, Thein PM, Junckerstorff R. Frailty, oral health and nutrition in geriatrics inpatients: a cross-sectional study. Gerodontology. 2019;36(3):223-28. doi: https://doi.org/10.1111/ger.12397

6. von Humboldt S, Leal I, Pimenta F. Living well in later life: the influence of sense of coherence, and socio-demographic, lifestyle and health-related factors on older adults' satisfaction with life. Appl Res Qual Life. 2014;9(3):631-42. doi: https://doi.org/10.1007/s11482-013-9262-6
7. Sewo Sampaio PY, Sampaio RA, Coelho Júnior HJ, Teixeira LF, Tessutti VD, Uchida MC, Arai H. Differences in lifestyle, physical performance and quality of life between frail and robust Brazilian community-dwelling elderly women. Geriatr Gerontol Int. 2016;16(7):829-35. doi: https://doi.org/10.1111/ ggi.12562

8. Lee SH, Kim YB. Which type of social activities may reduce cognitive decline in the elderly? a longitudinal population-based study. BMC Geriatr. 2016;16(1)165. doi: 10.1186/ s12877-016-0343-x

9. Schimmel M, Katsoulis J, Genton L, Müller F. Masticatory function and nutrition in old age. Swiss Dent J. 2015;125(4):449-54.

10. Lamster IB, Asadourian L, Del Carmen T, Friedman PK. The aging mouth: differentiating normal aging from disease. Periodontol 2000. 2016;72(1):96-107. doi: https://doi. org/10.1111/prd.12131

11. Emami E, Souza RF, Kabawat M, Feine JS. The impact of edentulism on oral and general health. Int J Dent. 2013;2013:498305. doi: https://doi.org/10.1155/2013/498305

12. Gil-Montoya JA, Mello AL, Barrios R, Gonzalez-Moles MA, Bravo M. Oral health in the elderly patient and its impact on general well-being: a nonsystematic review. Clin Interv Aging. 2015;10:461-7. doi: https://doi.org/ 10.2147/CIA.S54630

13. Scannapieco FA, Cantos A. Oral inflammation and infection, and chronic medical diseases: implications for the elderly. Periodontol 2000. 2016;72(1):153-75. doi: https://doi. org/10.1111/prd.12129

14. Kshetrimayum N, Reddy CV, Siddhana S, Manjunath M, Rudraswamy S, Sulavai S. Oral health-related quality of life and nutritional status of institutionalized elderly population aged 6o years and above in Mysore City, India. Gerodontology. 2013;30(2):119-25. doi: https://doi.org/10.1111/j. 1741-2358.2012.00651.x

15. Rodrigues JH, Marques MM, Biasotto-Gonzalez DA, Moreira MS, Bussadori SK, Mesquita-Ferrari RA, Martins MD. Evaluation of pain, jaw movements, and psychosocial factors in elderly individuals with temporomandibular disorder under laser phototherapy. Lasers Med Sci. 2015;30(3):953-9. doi: https://doi.org/10.1007/s10103-013-1514-z

16. Bernfort L, Gerdle B, Rahmqvist M, Husberg M, Levin LÅ. Severity of chronic pain in an elderly population in Sweden-impact on costs and quality of life. Pain. 2015;156(3):521-7. doi: https://doi.org/10.1097/01.j.pain.oooo460336.31600.01

17. Montgomery SC, Streit SM, Beebe ML, Maxwell PJ 4th. Micronutrient needs of the elderly. Nutr Clin Pract. 2014;29(4):43544. doi: https://doi.org/10.1177/o884533614537684 
18. Cruz-Jentoft AJ, Kiesswetter E, Drey M, Sieber CC. Nutrition, frailty, and sarcopenia. Aging Clin Exp Res. 2017;29(1):43-8. doi: https://doi.org/10.1007/s40520-016-0709-0

19. Naito M, Suzukamo Y, Nakayama T, Hamajima N, Fukuhara $\mathrm{S}$. Linguistic adaptation and validation of the general oral health assessment index (GOHAI) in an elderly Japanese population. J Public Health Dent. 2006;66(4):273-5. doi: https://doi.org/10.1111/j.1752-7325.2006.tb04081.x

20. Pinto VG. Saúde bucal coletiva. São Paulo: Santos; 2008.

21. Atchison KA, Dolan TA. Development of the geriatric oral health assessment index. J Dent Educ. 1990;54(11):680-7.

22. Campos JADB, Zucoloto ML, Bonafé FSS, Maroco J. General oral health assessment index: a new evaluation proposal. Gerodontology. 2017;34(3):334-42. doi: https://doi.org/ 10.1111/ger.12270

23. Hahnel S, Schwarz S, Zeman F, Schäfer L, Behr M. Prevalence of xerostomia and hyposalivation and their association with quality of life in elderly patients in dependence on dental status and prosthetic rehabilitation: a pilot study. J Dent. 2014;42(6):66470. doi: https://doi.org/10.1016/j.jdent.2014.03.003

24. Rebelo MA, Cardoso EM, Robinson PG, Vettore MV. Demographics, social position, dental status and oral health-related quality of life in community-dwelling older adults. Qual Life Res. 2016;25(7):1735-42. doi: 10.1007/s11136-015-1209-y

25. Sergi G, Bano G, Pizzato S, Veronese N, Manzato E. Taste loss in the elderly: Possible implications for dietary habits. Crit Rev Food Sci Nutr. 2017;57(17):3684-9. doi: https://doi. org/10.1080/10408398.2016.1160208

26. Ikebe K, Matsuda K, Kagawa R, Enoki K, Okada T, Yoshida M, Maeda Y. Masticatory performance in older subjects with varying degrees of tooth loss. J Dent. 2012;40(1):71-6. doi: https://doi.org/10.1016/j.jdent.2011.10.007

27. Avivi-Arber L, Sessle BJ. Jaw sensorimotor control in healthy adults and effects of ageing. J Oral Rehabil. 2018;45(1):50-8o. doi: https://doi.org/10.1111/joor.12554

28. Moynihan P, Thomason M, Walls A, Gray-Donald K, Morais JA, Ghanem H, et al. Researching theimpact of oral health on diet and nutritional status: methodological issues. J Dent. 2009;37(4): 237-49. doi: https://doi.org/10.1016/j.jdent.2008.12.003

29. Sato N, Ono T, Kon H, Sakurai N, Kohno S, Yoshihara A, Miyazaki H. Ten-year longitudinal study on the state of dentition and subjective masticatory ability in community-dwelling elderly people. J Prosthodont Res. 2016;60(3):17784. doi: 10.1016/j.jpor.2015.12.008

30. Lavigne GJ, Sessle BJ. The neurobiology of orofacial pain and sleep and their interactions. J Dent Res. 2016;95(10):110916. doi: https://doi.org/10.1177/o022034516648264
31. Iinuma T, Hirata T, Arai Y, Takayama M, Abe Y, Fukumoto $\mathrm{M}$, et al. Perceived swallowing problems and mortality risk in very elderly people $\geq 85$ years old: Results of the Tokyo Oldest Old Survey on Total Health study. Gerodontology. 2017;34(3):313-9. doi: https://doi.org/10.1111/ger.12265

32. Albaker AM. The oral health-related quality of life in edentulous patients treated with conventional complete dentures. Gerodontology. 2013;30(1):61-6. doi: https://doi. org/10.1111/j.1741-2358.2012.00645.x

33. Roy N, Kim J, Courey M, Cohen SM. Voice disorders in the elderly: a national database study. Laryngoscope. 2016;126(2):421-8. doi: https://doi.org/10.1002/lary.25511

34. Kim J, Nam C, Kim Y, Kim H. The development of the Geriatric Index of Communicative Ability (GICA) for measuring communicative competence of elderly: A pilot study. Speech Commun. 2014;56:63-9. doi: https://doi.org/10.1016/j.specom.2013.08.001

35. Amarya S; Singh K, Sabharwal M. Changes during aging and their association with malnutrition. J Clin Gerontol Geriatr. 2015;6(3):78-84. doi: https://doi.org/10.1016/j.jcgg.2015.05.003 36. Silva AE, Demarco FF, Feldens CA. Oral health-related quality of life and associated factors in Southern Brazilian elderly. Gerodontology. 2015;32(1):35-45. doi: https://doi. org/10.1111/ger.12050

37. Amagai N, Komagamine Y, Kanazawa M, Iwaki M, Jo A, Suzuki H, Minakuchi S. The effect of prosthetic rehabilitation and simple dietary counseling on food intake and oral health related quality of life among the edentulous individuals: a randomized controlled trial. J Dent. 2017;65:89-94. doi: https://doi.org/10.1016/j.jdent.2017.07.011

38. Andersson K, Nordenram G. Attitudes to and perceptions of oral health and oral care among community-dwelling elderly residents of Stockholm, Sweden: an interview study. Int J Dent Hyg. 2004;2(1):8-18. doi: https://doi.org/10.1111/j. 1601-5037.2004.00059.x

39. Lajnert V, Kovacevic Pavicic D, Pavlic A, Pokrajac-Bulian A, Spalj S. Smile Aesthetics Satisfaction Scale: development and validation of a new brief five-item measure of satisfaction with smile aesthetics in adults and the elderly. Int Dent J. 2018;68(3):162-70. doi: https://doi.org/10.1111/idj.12362 40. Melo LA, Sousa MM, Medeiros AKB, Carreiro AFP, Lima KC. Factors associated with negative self-perception of oral health among institutionalized elderly. Ciênc Saúde Colet. 2016;21(11):3339-46. doi: https://doi.org/10.1590/1413812320152111.08802015

41. Echeverria MS, Wünsch IS, Langlois CO, Cascaes AM, Ribeiro Silva AE. Oral health-related quality of life in older adul- 
ts-Longitudinal study. Gerodontology. 2019;36(2):118-24. Epub 2018 Dec 18. doi: https://doi.org/10.1111/ger.12387 42. Cavalera C, Pagnini F, Zurloni V, Diana B, Realdon O, Castelnuovo $\mathrm{G}$, et al. Shame proneness and eating disorders: a comparison between clinical and non-clinical samples. Eat Weight Disord-St. 2016;21(4):701-7. doi: https://doi.org/ 10.1007/s40519-016-0328-y

43. Lindroos EK, Saarela RK, Suominen MH, Muurinen S, Soini H, Kautiainen H, et al. Burden of Oral Symptoms and Its Associations With Nutrition, Well-Being, and Survival Among Nursing
Home Residents. J Am Med Dir Assoc. 2018;20(5):537-43. doi: https://doi.org/ 10.1016/j.jamda.2018.10.025

44. Takiguchi T, Yoshihara A, Takano N, Miyazaki H. Oral health and depression in older Japanese people. Gerodontology. 2016;33(4):439-46. doi: https://doi.org/10.1111/ger.12177

45. Durham J, Fraser HM, McCracken GI, Stone KM, John MT, Preshaw PM. Impact of periodontitis on oral health-related quality of life. J Dent. 2013;41(4):370-6. doi: https://doi. org/10.1016/j.jdent.2013.01.008 